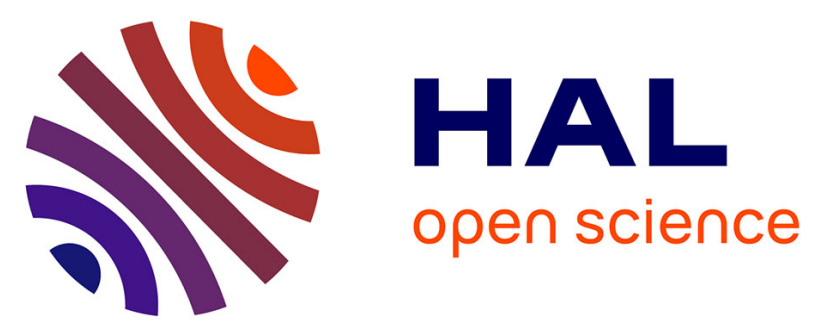

\title{
Analysis of the non-linearity of the heat transfer equation in case of a time-dependent heat source: application to the 3 omega method
}

\author{
T. Ding, Yves Jannot, Vincent Schick, Alain Degiovanni
}

\section{- To cite this version:}

T. Ding, Yves Jannot, Vincent Schick, Alain Degiovanni. Analysis of the non-linearity of the heat transfer equation in case of a time-dependent heat source: application to the 3 omega method. Journal of Engineering Mathematics, 2020, 121 (1), pp.85-99. 10.1007/s10665-020-10040-z . hal-02861750

\section{HAL Id: hal-02861750 \\ https://hal.univ-lorraine.fr/hal-02861750}

Submitted on 9 Jun 2020

HAL is a multi-disciplinary open access archive for the deposit and dissemination of scientific research documents, whether they are published or not. The documents may come from teaching and research institutions in France or abroad, or from public or private research centers.
L'archive ouverte pluridisciplinaire HAL, est destinée au dépôt et à la diffusion de documents scientifiques de niveau recherche, publiés ou non, émanant des établissements d'enseignement et de recherche français ou étrangers, des laboratoires publics ou privés. 
archives-ouvertes

\section{Analysis of the non-linearity of the heat transfer equation in case of a time- dependent heat source: application to the 3 omega method}

T. Ding, Yves Jannot, Vincent Schick, Alain Degiovanni, Al Jadida

\section{- To cite this version:}

T. Ding, Yves Jannot, Vincent Schick, Alain Degiovanni, Al Jadida. Analysis of the non-linearity of the heat transfer equation in case of a time- dependent heat source: application to the 3 omega method. Article in Journal of Engineering Mathematics, 2020, 121 (1), pp.85-99. 10.1007/s10665-020-10040-z . hal-02861750

\section{HAL Id: hal-02861750 \\ https://hal.univ-lorraine.fr/hal-02861750}

Submitted on 9 Jun 2020

HAL is a multi-disciplinary open access archive for the deposit and dissemination of scientific research documents, whether they are published or not. The documents may come from teaching and research institutions in France or abroad, or from public or private research centers.
L'archive ouverte pluridisciplinaire HAL, est destinée au dépôt et à la diffusion de documents scientifiques de niveau recherche, publiés ou non, émanant des établissements d'enseignement et de recherche français ou étrangers, des laboratoires publics ou privés. 


\title{
Analysis of the non-linearity of the heat transfer equation in case of a time-dependent heat source: application to the $3 \omega$ method $^{1}$
}

\author{
T. Ding ${ }^{1}$, Y. Jannot ${ }^{1}$, V. Schick ${ }^{1}$, A. Degiovanni ${ }^{1,2}$ \\ ${ }^{1}$ Université de Lorraine, CNRS, LEMTA, F-54500 Vandœuvre-lès-Nancy, France \\ ${ }^{2}$ Université Internationale de Rabat, Pôle Energie, LERMA, Rocade Rabat-Salé, 11100, Sala \\ Al Jadida, Morocco
}

\begin{abstract}
The $3 \omega$ method may be used to estimate the thermal conductivity of an electric conducting wire. In this method, an alternating voltage with an angular frequency $\omega$ is applied to the wire. The resulting low tension $U_{3 \omega}$ of angular frequency $3 \omega$ that appears in the total tension is extracted by a lock-in amplifier. The amplitude of $U_{3 \omega}$ is directly linked to the thermal conductivity of the wire and enables its estimation. All authors using the $3 \omega$ method for the determination of the thermal conductivity of an electric conducting wire considered that the heat flux produced by Joule effect in the wire is constant. This hypothesis leads to a linear form of the heat transfer equation. In this paper, an analytical model taking into account the dependence of the heat flux on the temperature is developed, it leads to a non-linear form of the heat transfer equation. The importance of the non-linearity in certain cases is demonstrated and the analytical solution is used to define a unique criterion that must be verified to ensure the validity of the linear solution.
\end{abstract}

Keywords: thermal conductivity, non-linearity, $3 \omega$ method, transient method, wire

\section{Introduction}

During the last decades the $3 \omega$ method became one of the most popular methods to measure the thermal conductivity of the thin layers. Its origin goes back to the works of Corbino [1] who detected a low tension of angular frequency $3 \omega$ when he applied an alternating voltage of angular frequency $\omega$ to a resistance. This phenomenon was successively used to measure the calorific capacity of the heating element itself and then to measure the calorific capacity of a substrate by using a wide ribbon and a 1D model of heat transfer [2-7]. The method such as it is presently known results from the works of Cahill who proposed a 2D analytical model which can apply to a thin strip [8]. This allows the measurement of the thermal conductivity of low thickness layers [9-10].

Several authors [11-18] have then adapted and used this method initially developed to characterize flat thin layers for the thermal characterization of electrical conductive wires of micrometric and even nanometric diameter [19].

$\mathrm{Lu}$ and al [11] presents a 1D model in which the convective and radiation transfers on the surface of the wire are neglected. They deduce an expression of the component $U_{3 \omega}$ with

\footnotetext{
${ }^{1}$ Ding, T., Jannot, Y., Schick, V. et al. Analysis of the non-linearity of the heat transfer equation in case of a time-dependent heat source: application to the $3 \omega$ method. J Eng Math (2020). https://doi.org/10.1007/s10665$020-10040-\mathrm{z}$
} 
angular frequency $3 \omega$ of the measured tension in the form of an infinite sum. They show then that for low frequencies, the first term of this sum is sufficient to obtain a satisfying precision. Dames and Chen [12] use the same 1D model and present another expression of the average temperature of the wire. They deduce a simplified expression of $U_{3 \omega}$ close to the relation obtained by Lu and al [11].

The models and the methods proposed by Lu and al [11] and Dames and Chen [12] were then used by other authors to realize the thermal characterization of wires with different composition and dimensions: carbon fiber of diameter $7 \mu \mathrm{m}$ and of length between 0.8 and $1.9 \mathrm{~mm}$ [13], platinum wire of diameter $25 \mu \mathrm{m}$ [14], bismuth tellurure wire of diameter $180 \mathrm{~nm}$ and of length $4 \mu \mathrm{m}$ [15 ], nickel wire of diameter $150 \mathrm{~nm}$ and of length $5.44 \mu \mathrm{m}$ [16], nanowire of carbon of diameter $45 \mathrm{~nm}$ [17], silicon wire of diameter $150 \mathrm{~nm}$ and of length $5-10 \mu \mathrm{m}$ [18].

Nevertheless, all the authors, Depasse et al [20] excepted, made the hypothesis that the heat flux produced in the wire by Joule effect is constant. But in most experiments the current intensity is kept constant so that the heat flux is not constant because the wire electric resistance varies with the temperature. In this case, the system of equations satisfied by the temperature is no longer linear.

The object of the presented work is to establish an expression of $U_{3 \omega}$ based on a model taking into account the radiation and the convective losses, the heat transfer between the connecting wires and the wire to be characterized, as well as the non-linear heat source.

In this paper the classical device and the principle of the $3 \omega$ method will first be detailed. The expression of the mean temperature rising of the wire, considering a constant heat flux but taking into account the connecting wires, will be established. Then a more complete model taking into account the variation of the heat flux will be developed.

A sensitivity study of the wire mean temperature to the thermal parameters to be estimated will be undertaken for various dimensions and thermal properties of the wire. The importance of the non-linearity of the heat source in certain conditions will be demonstrated and the model simulation will be compared to experimental results obtained on a micrometric wire.

\section{Materials and methods}

Figure 1 represents the $3 \omega$ device generally used to measure the thermal properties of an electrical conductive material which electric resistance varies linearly with the temperature. The low diameter wire is welded to higher diameter connecting wires. A high value of the ratio between the diameter of the connecting wires and the diameter of the wire to be measured ensures that the temperature of the welds remains constant during the measure. The validity of this hypothesis will be further discussed. The temperature is assumed to be uniform in a crosssection of the wire since its diameter is low.

A sinusoidal current with intensity $I=I_{0} \cos (\omega t)$ passes through the wire so that a heat flow rate is generated in it by Joule effect. This heat flow rate $P$ is generally considered as constant:

$P=R I^{2} \approx R_{0} I_{0}^{2} \cos ^{2}(\omega t)=\frac{R_{0} I_{0}^{2}}{2}[1+\cos (2 \omega t)]=P_{D C}+P_{A C}$,

with:

$R_{0} \quad$ Wire electrical resistance at $T_{\text {ext }}(\Omega)$

$R \quad$ Wire electrical resistance at temperature $T(\Omega)$ 


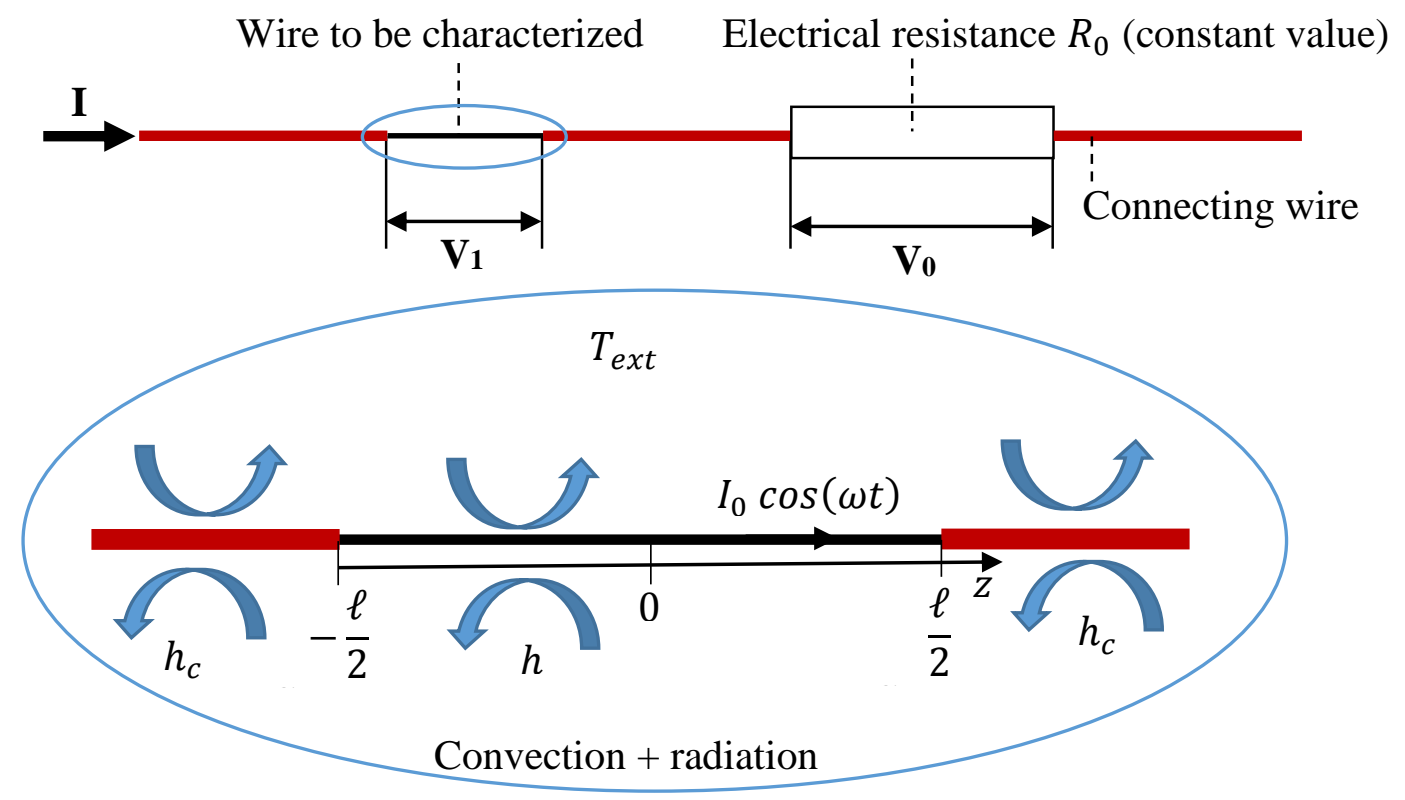

Figure 1: Schema of the measurement device

It is the sum of a constant part $P_{D C}=R_{0} I_{0}{ }^{2} / 2$ and of a sinusoidal part $P_{A C}=$ $\left(R_{0} I_{0}{ }^{2} / 2\right) \cos (2 \omega t)$. The mean temperature rise is proportional to the heat flow rate, thus it contains a constant part and a sinusoidal part:

$\Delta T(t)=\bar{T}-T_{e x t}=\Delta T_{D C}+\Delta T_{A C} \cos (2 \omega t+\varphi)$,

where $\bar{T}$ is the wire mean temperature along $O x$ (cf. Fig.1) and $\Delta T_{D C}$ and $\Delta T_{A C}$ are the two mean temperature amplitudes due respectively to the continuous and sinusoidal parts of the heat flow rate. $\varphi$ is the phase shift between the current and the temperature.

Since the wire resistance varies linearly with the temperature, it can be written as:

$R(t)=R_{0}(1+\alpha \Delta T)=R_{0}\left[1+\alpha \Delta T_{D C}+\alpha \Delta T_{A C} \cos (2 \omega t+\varphi)\right]$,

with:

$\alpha \quad$ Temperature coefficient of the electric resistance $\left(\mathrm{K}^{-1}\right)$

The electrical potential difference $U=V_{1}-V_{0}$ (cf. figure 1) can be expressed by the formula:

$U=R_{0} I_{0}\left(1+\alpha \Delta T_{D C}\right) \cos (\omega t)+\frac{R_{0} I_{0}}{2} \alpha \Delta T_{A C} \cos (\omega t+\varphi)+\frac{R_{0} I_{0}}{2} \alpha \Delta T_{A C} \cos (3 \omega t+\varphi)$.

The third harmonic $U_{3 \omega}$ of $U$ is measured and used to estimate the thermal conductivity $\lambda$ of the wire because it only contains one term (contrarily to the first harmonic $U_{1 \omega}$ ), directly proportional to the amplitude $\Delta T_{A C}$ of the mean temperature rise:

$U_{3 \omega}(t)=\frac{1}{2} R_{0} I_{0} \alpha \Delta T_{A C} \cos (3 \omega t+\varphi)$.

A complete model of the amplitude $\Delta T_{A C}$ will now be developed and then used to calculate its sensitivity to the different characteristics of the wire.

\section{Steady periodic regime with constant heat flux}

In this section, the case of the steady periodic regime with a constant heat flux is considered, and the influence of the connecting wires will be studied. 


\subsection{Analytical model}

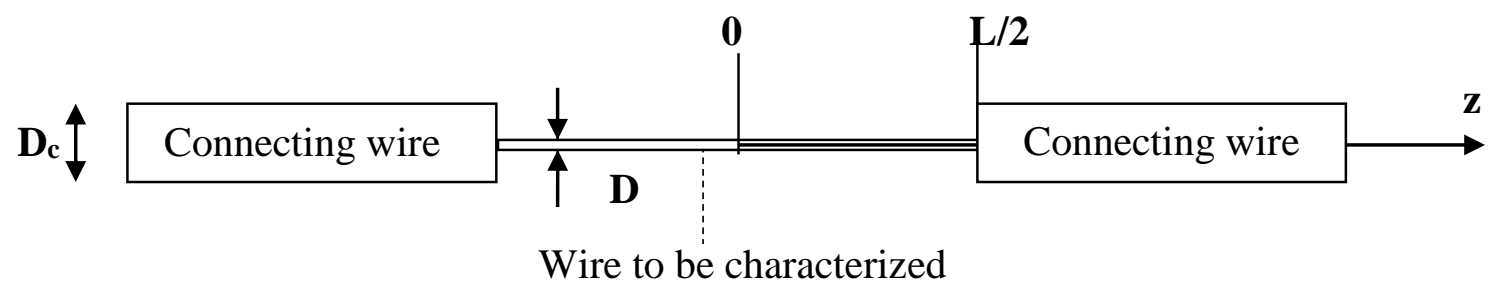

Figure 2: Geometrical characteristics

The wire temperature $T$ and the connecting wire temperature $T_{c}$ verify the following equations (cf. figure 2):

Heat equation for the wire:

$\frac{\partial^{2} T}{\partial z^{2}}-\frac{h m}{\lambda S}\left(T-T_{\text {ext }}\right)+\frac{\dot{q}}{\lambda}=\frac{1}{a} \frac{\partial T}{\partial t}, \quad$ for $0<z<\ell / 2$,

with: $m=\pi D, S=\frac{\pi D^{2}}{4}$ and $\dot{q}=\frac{R I^{2}}{S \ell}$,

where $h$ is the heat transfer coefficient $\left(\mathrm{W} \mathrm{m}^{-2} \mathrm{~K}^{-1}\right)$ on the perimeter of the wire, $a$ the thermal diffusivity $\left(\mathrm{m}^{2} \mathrm{~s}^{-1}\right)$ of the wire and $\lambda$ its thermal conductivity $\left(\mathrm{W} \mathrm{m}^{-1} \mathrm{~K}^{-1}\right)$.

Heat equation for the connecting wires:

$\frac{\partial^{2} T}{\partial z^{2}}-\frac{h_{c} m_{c}}{\lambda_{c} S_{c}}\left(T_{c}-T_{\text {ext }}\right)=\frac{1}{a_{c}} \frac{\partial T_{c}}{\partial t}, \quad$ for $\ell / 2<z<\infty$,

with: $m_{c}=\pi D_{c}$ and $S_{c}=\frac{\pi D_{c}{ }^{2}}{4}$,

where $h_{c}$ is the heat transfer coefficient $\left(\mathrm{W} \mathrm{m}^{-2} \mathrm{~K}^{-1}\right)$ on the perimeter of the connecting wire, $a_{c}$ the thermal diffusivity $\left(\mathrm{m}^{2} \mathrm{~s}^{-1}\right)$ of the connecting wire and $\lambda_{c}$ its thermal conductivity ( $\mathrm{W} \mathrm{m}^{-}$ $\left.{ }^{1} \mathrm{~K}^{-1}\right)$.

For symmetry reason, the temperature reaches an extremum at the center of the wire:

$\frac{\partial T}{\partial z}=0, \quad$ for $z=0$.

The connecting wires are considered as semi-infinite media:

$T_{c} \rightarrow T_{\text {ext }}$,

for $z \rightarrow \infty$.

The continuity of the temperature at the welding point leads to:

$T=T_{c}, \quad$ for $z=\ell / 2$.

The continuity of the heat flux assuming a purely 1D transfer in the $\mathrm{Oz}$ direction (the constriction is neglected) leads to:

$\lambda S \frac{\partial T}{\partial z}=\lambda_{c} S_{c} \frac{\partial T_{c}}{\partial z}$

for $z=\ell / 2$

The initial condition can be written as:

$T=T_{c}=T_{\text {ext }}, \quad$ at $t=0$.

At first, it will be considered that the resistance $R$ is very close to $R_{0}$ when calculating the volume heat flow rate $\left(\mathrm{W} \mathrm{m}^{-3}\right)$ produced by Joule effect:

$$
\dot{q} \approx \frac{R_{0} I^{2}}{S \ell}=\frac{4 R_{0} I_{0}{ }^{2} \cos ^{2}(\omega t)}{\pi D^{2} \ell}=\frac{4 R_{0} I_{0}^{2}}{\pi D^{2} \ell} \frac{1}{2}[1+\cos (2 \omega t)] .
$$

This approximation is justified by the low value of the temperature coefficient of the wire: $\alpha \ll 1$. 
The heat source can be considered as the sum of a flux step and of a periodic flux with an angular frequency $2 \omega$. If a flux proportional to $\sin (2 \omega t)$ is associated to the periodic flux proportional to $\cos (2 \omega t)$, the periodic heat source may be written as:

$$
\dot{q}_{A C}=\frac{2 R_{0} I_{0}^{2}}{\pi D^{2} \ell} \exp (2 \mathrm{i} \omega t)
$$

Writing the periodic solution as: $T-T_{\text {ext }}=\psi(z, \omega) \exp (2 \mathrm{i} \omega t)$ and: $\quad T_{c}-T_{\text {ext }}=$ $\psi_{c}(z, \omega) \exp (2 i \omega t)$, the equations become:

$\frac{\mathrm{d}^{2} \psi}{\mathrm{d} z^{2}}+K_{1}-\beta^{2} \psi=0$

$$
\begin{aligned}
& 0<z<\ell / 2 \\
& \ell / 2<z<\infty
\end{aligned}
$$

$\frac{\mathrm{d}^{2} \psi_{c}}{\mathrm{~d} z^{2}}-\beta_{c}{ }^{2} \psi=0$,

$\frac{\mathrm{d} \psi}{\mathrm{d} z}=0$,

for $z=0$,

$\psi_{c}=0$,

for $z \rightarrow \infty$,

$\psi=\psi_{c}$,

for $z=\ell / 2$,

$\lambda D^{2} \frac{\mathrm{d} \psi}{\mathrm{d} z}=\lambda_{c} D_{c}{ }^{2} \frac{\mathrm{d} \psi_{c}}{\mathrm{~d} z}$,

for $z=\ell / 2$,

with:

$K_{1}=\frac{2 R_{0} I_{0}^{2}}{\pi D^{2} \ell \lambda}$,

$\beta^{2}=\frac{2 \mathrm{i} \omega}{a}+\frac{4 h}{\lambda D}$,

$\beta_{c}{ }^{2}=\frac{2 \mathrm{i} \omega}{a_{c}}+\frac{4 h_{c}}{\lambda_{c} D_{c}}$.

The general solutions are:

$\psi(z, \omega)=A \cosh (\beta z)+B \sinh (\beta z)+\frac{K_{1}}{\beta^{2}}$,

$\psi_{c}(z, \omega)=A_{c} \cosh \left(\beta_{c} z\right)+B_{c} \sinh \left(\beta_{c} z\right)$.

Using relations (17) to (20) leads to the expression of the wire temperature:

$T-T_{\text {ext }}=\psi(z, \omega) \exp (2 \mathrm{i} \omega t)=\frac{K_{1}}{\beta^{2}}\left[1-\frac{\cosh (\beta z)}{\cosh \left(\beta \frac{\ell}{2}\right)+\frac{D^{2} \lambda \beta}{D_{c} \lambda_{c} \beta_{c}} \sinh \left(\beta \frac{\ell}{2}\right)}\right] \exp (2 \mathrm{i} \omega t)$,

and the wire mean temperature can be written:

$\bar{T}-T_{\text {ext }}=\bar{\psi}(\omega) \exp (2 \mathrm{i} \omega t)=\frac{K_{1}}{\beta^{2}}\left[1+\frac{2[1-\cosh (\beta \ell)]}{\beta \ell\left\{\sinh (\beta \ell)-\frac{D^{2} \lambda \beta}{D_{c}^{2} \lambda_{c} \beta_{c}}[1-\cosh (\beta \ell)]\right\}}\right] \exp (2 \mathrm{i} \omega t)$.

The amplitude $\Delta T_{A C}$ of the mean periodic temperature rise is the amplitude of $\bar{\psi}(\omega)$.

If the wire extremities can be considered at constant temperature (if: $D^{2} \lambda \beta / D_{c}{ }^{2} \lambda_{c} \beta_{c} \ll 1$ ), relation (27) becomes:

$\bar{\psi}(\omega)=\frac{K_{1}}{\beta^{2}}\left[1+\frac{2[1-\cosh (\beta \ell)]}{\beta \ell \sinh (\beta \ell)}\right]$.

This last relation is identical to that established by [21].

\subsection{Influence of the connection wires}

Three different wires made of Chromel, copper and iron having thus very different thermal and 
thermoelectric properties (thermal conductivity, electric resistivity and temperature coefficient) will be considered as an example.
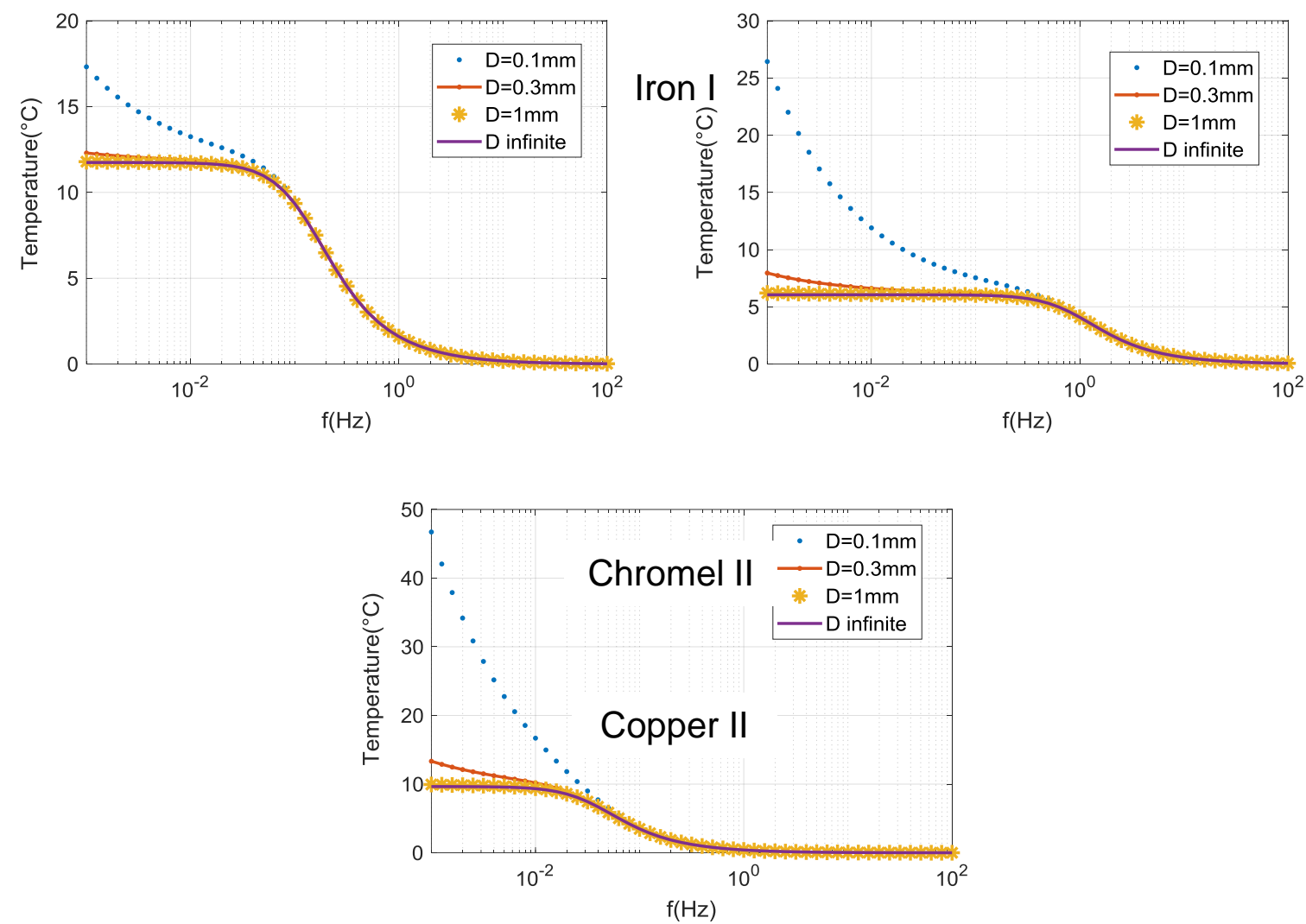

Figure 3: Amplitude $\Delta T_{A C}$ of the mean wire temperature as a function of the frequency $f$ for three wires: "Iron I", "Copper II" and "Chromel II"

The properties of each wire are given in table 1 and the temperature evolution will be simulated for the conditions "Iron I", "Copper II" and "Chromel II" described in table 2. The following diameters of the connection copper wires will be considered successively: $100 \mu \mathrm{m}, 300 \mu \mathrm{m}$ and $1 \mathrm{~mm}$. Figures 3 represent the calculated amplitude of the temperature, which is directly proportional to $U_{3 \omega}$ according to relation (5), as a function of the current angular frequency $\omega$. It can be seen that the connection wires have a great influence if their diameters are $100 \mu \mathrm{m}$. But, whatever are the characteristics of the wire to be characterized, the connection wires have not any influence on the electric potential $U_{3 \omega}$ if their diameters are greater than $1 \mathrm{~mm}$. In this case the problem may be simplified and it may be considered that the temperatures of the extremities of the wire are constant as boundary conditions.

This conclusion may appear in contradiction with the results of Hou [22] who have demonstrated that the connecting wires have no influence on the mean wire temperature. However, he considered the connecting wires as semi-infinite media while we considered them as cylinders exchanging heat by convection and radiation with the environment.

\section{Steady periodic regime with variable heat flux}

In this section the influence of the variation of the heat flux (previously neglected) will be studied. It corresponds to the practical case where the intensity is kept constant. 
As it will be shown, taking into account the connection wires does not change the temperature expression if their diameters are sufficiently high. Thus, for the complete calculation the hypothesis of a constant temperature (equal to the ambient temperature $T_{\text {ext }}$ ) for the extremities of the wire will be considered.

If the non-linearity is taken into account, the number of harmonics of the steady periodic regime is infinite. It is then more interesting to solve the problem in the Laplace space, rather than in the Fourier space as done in [20].

\subsection{Analytical model}

The governing equations of the heat transfer are:

$\frac{\partial^{2} T^{*}}{\partial z^{2}}-\frac{4 h}{\lambda S} T^{*}+\frac{\dot{q}}{\lambda}=\frac{1}{a} \frac{\partial T^{*}}{\partial t}$,

with: $T^{*}=T-T_{\text {ext }}$,

$\frac{\partial T^{*}}{\partial z}=0$,

$$
\begin{aligned}
& \text { for } z=0, \\
& \text { for } z=\ell / 2, \\
& \text { at } t=0,
\end{aligned}
$$

$T^{*}=0$,

with: $\dot{q}=\frac{R I^{2}}{S \ell}=\frac{4 R_{0}(1+\alpha T) I_{0}^{2} \cos ^{2}(\omega t)}{\pi D^{2} \ell}$ (variable with the temperature).

The relation (29) becomes:

$\frac{\partial^{2} T^{*}}{\partial z^{2}}-K_{2} T^{*}+K_{3} f(t)+\alpha K_{3} T^{*} f(t)=\frac{1}{a} \frac{\partial T^{*}}{\partial t}$,

with: $K_{2}=\frac{4 h}{\lambda D}$,

and $\quad K_{3}=\frac{4 R_{0} I_{0}{ }^{2}}{\pi D^{2} \lambda \ell}$.

The system is non-linear because of the term $\alpha K_{3} T^{*} f(t)$.

Given that:

$\frac{1}{a} \frac{\partial T^{*}}{\partial t}-\alpha K_{3} T^{*} f(t)=\frac{1}{a} \exp \left[\alpha K_{3} a \int f(t) \mathrm{d} t\right] \frac{\partial}{\partial t}\left\{T^{*} \exp \left[-\alpha K_{3} a \int f(t) \mathrm{d} t\right]\right\}$,

one can define $F$ as: $F=T^{*} \exp \left[-\alpha K_{3} a \int f(t) \mathrm{d} t\right]$,

and relation (34) becomes:

$\frac{\partial^{2} F}{\partial z^{2}}-K_{2} F+K_{3} f(t) \exp \left[-\alpha K_{3} a \int f(t) \mathrm{d} t\right]=\frac{1}{a} \frac{\partial F}{\partial t}$.

Relation (39) is a linear partial differential equation with a source $g(t)$ given by:

$$
g(t)=K_{3} f(t) \exp \left[-\alpha K_{3} a \int f(t) \mathrm{d} t\right] .
$$

This is a classical problem that can be solved using the Green method [23]. The source $g(t)$ is replaced by the Dirac function $\delta$ at time $t=0$, thus :

$\frac{\partial^{2} G}{\partial z^{2}}-K_{2} G+\delta(t=0)=\frac{1}{a} \frac{\partial G}{\partial t}$,

$G$ is the Green function of the problem. 
The solution $F$ of equation (39) is given by a convolution product:

$F=G \otimes g(t)$.

To solve equation (40) a Laplace transformation is applied, leading to:

$\frac{\mathrm{d}^{2} \theta}{\mathrm{d} z^{2}}-\beta^{2} \theta+1=0$,

with: $\beta^{2}=\frac{p}{a}+\frac{4 h}{\lambda D}$,

and $\theta=\mathcal{L}(G)=\int_{0}^{\infty} G(t) \exp (-p t) \mathrm{d} t$.

The solution is:

$\theta(p, z)=\frac{1}{\beta^{2}}\left[1-\frac{\cosh (\beta z)}{\cosh \left(\beta \frac{\ell}{2}\right)}\right]$,

and the mean temperature can be written as:

$\bar{\theta}(p)=\frac{1}{\beta^{2}}\left\{1+\frac{2[1-\cosh (\beta \ell)]}{\beta \ell \sinh (\beta \ell)}\right\}$.

Then: $\bar{G}=\mathcal{L}^{-1}(\bar{\theta})$,

and applying the Green method: $\bar{F}=\mathcal{L}^{-1}(\bar{\theta}) \otimes g(t)$,

where $\bar{F}$ is the mean value of $F$.

So that the general solution is:

$\bar{T}^{*}(t)=\exp \left[\alpha K_{3} a \int f(t) \mathrm{d} t\right]\left[\mathcal{L}^{-1}(\bar{\theta}) \otimes g(t)\right]$,

with: $\bar{T}^{*}=\bar{T}-T_{\text {ext }}$.

In our case: $f(t)=\cos ^{2}(\omega t)$,

and: $g(t)=K_{3} \cos ^{2}(\omega t) \exp \left\{-\alpha K_{3} a \frac{1}{2}\left[t+\frac{\sin (2 \omega t)}{2 \omega}\right]\right\}$.

Thus: $\bar{T}^{*}(t)=\exp \left\{\alpha K_{3} a \frac{1}{2}\left[t+\frac{\sin (2 \omega t)}{2 \omega}\right]\right\}\left[\mathcal{L}^{-1}(\bar{\theta}) \otimes g(t)\right]$,

and the measured tension is:

$U(t)=V_{1}(t)-V_{0}=R_{0}\left[1+\alpha\left(\bar{T}-T_{\text {ext }}\right)\right] I-R_{0} I=\bar{T}^{*} \alpha R_{0} I_{0} \cos (\omega t)$.

\subsection{Sensitivity analysis}

A sensitivity study has been realized for three wires placed under vacuum, i.e. with a weak heat transfer coefficient corresponding to radiation heat transfer $\left(h=3 \mathrm{~W} \mathrm{~m}^{-2} \mathrm{~K}^{-1}\right)$ and for another iron wire under atmospheric pressure with a higher heat transfer coefficient $(h=$ $500 \mathrm{~W} \mathrm{~m}^{-2} \mathrm{~K}^{-1}$ ). These four cases correspond to the configurations «Iron V », «Iron II », «Copper IV» and «Chromel IV» described in table 2 and the corresponding results are reported in figure 4.

These figures represent the reduced sensitivities of the amplitude $\Delta T_{A C}$ of the wire mean periodic temperature to the thermal conductivity $\lambda$, the volume heat capacity $\rho c$ and the heat transfer coefficient $h: \lambda \frac{\partial\left(\Delta T_{A C}\right)}{\partial \lambda}, \rho c \frac{\partial\left(\Delta T_{A C}\right)}{\partial \rho c}$ and $h \frac{\partial\left(\Delta T_{A C}\right)}{\partial h}$. 

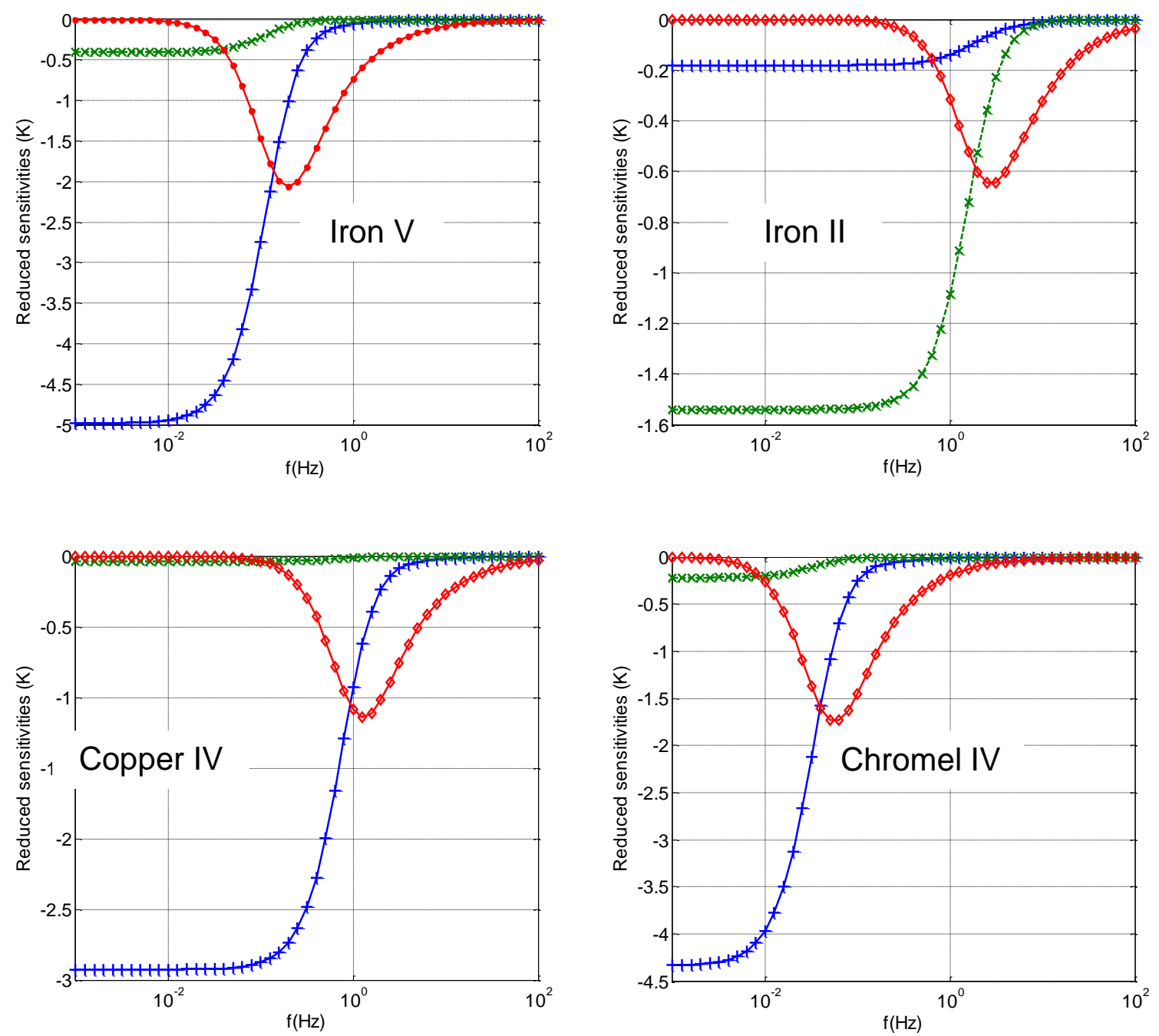

Figure 4: Reduced sensitivities of the wire mean temperature amplitude $\Delta T_{A C}$ to $\lambda(+), \rho c(\diamond)$ and $h(\boldsymbol{X})$ for "Iron V", "Iron II", "Copper IV" and "Chromel IV" (cf. Table 2)

It can be seen that for all wires the sensitivity to the thermal conductivity is correlated to the sensitivity to the heat transfer coefficient. Thus the experiments must be realized under vacuum to minimize the influence of $h$. On the contrary, this technique could be used to measure the heat transfer coefficient if the thermal conductivity of the wire is known; preliminary tests have demonstrated the feasibility of this technique.

The sensitivity to $\rho c$ is not correlated to the two other sensitivities since it is the only one to reach an extremum so that an estimation of $\rho c$ would be possible for each case.

\section{Results}

At first, the linear (constant heat flux) and the non-linear (variable heat flux) solutions will be compared for the three previously described wires. The solution of relation (54) is obtained using the IFFT (Inverse Fast Fourier Transform) algorithm (Matlab function "ifft") to realize the inverse Laplace transformation. The solution is programmed with Matlab and the amplitude of the $3 \omega$ component is obtained by FFT (Matlab function "fft") after the convolution product (Matlab function "conv") has been realized. As an example, figure 5 represents the amplitude 
of the electrical tension $U_{3 \omega}^{\ell}$ as a function of the electrical current frequency for the configurations "Iron I" and "Copper I" when considering a constant heat flux (linear solution) and a variable heat flux (non-linear solution).
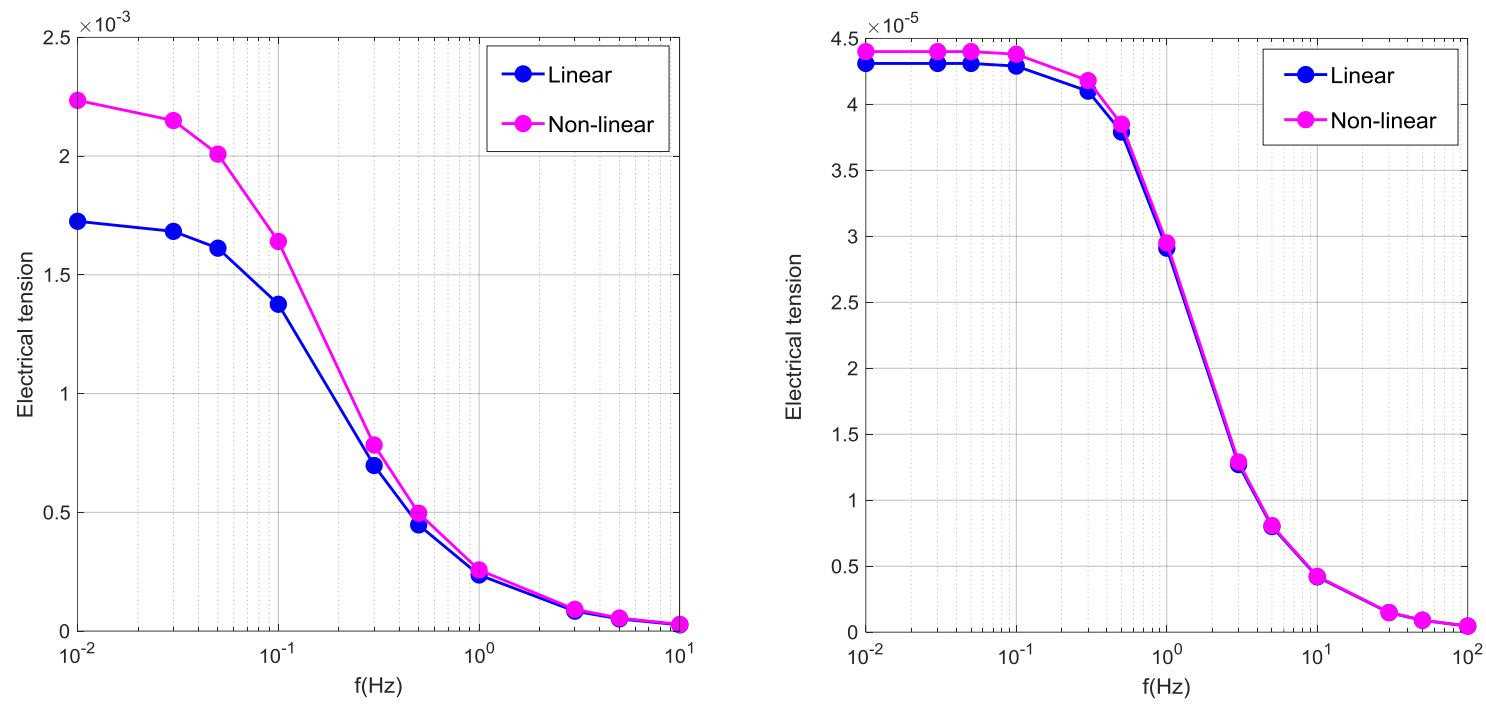

Figure 5: Amplitude of the electrical tension $U_{3 \omega}$ as a function of the electrical current frequency for the configurations "Iron I" and "Copper I" with linear and non-linear hypothesis

The deviation between the linear and the non-linear solutions may reach high values up to $30 \%$ for Iron for example.

Since the non-linear calculation of the amplitude of the electrical tension $U_{3 \omega}$ is quite complex (relation (54)), it is difficult to use it to estimate the thermal conductivity of the wire by an inverse method. The best solution seems to choose experimental conditions so that deviation between the linear and the non-linear solutions is negligible, enabling the use of the easy to run linear solution. To do this, the maximum relative deviations for fifteen different configurations described in table 2 have been calculated and relations (28) to (54) have been re-written under a non-dimensional form.

The amplitude of $U_{3 \omega}$ given by relation (28) depends on one non-dimensional number that is:

$\gamma_{2}=\frac{4 h \ell^{2}}{\lambda D}$.

So that: $U_{3 \omega}^{\ell *}=f_{\ell}\left(\gamma_{2}, \omega^{*}\right)$,

with: $U_{3 \omega}^{\ell *}=\frac{U_{3 \omega}^{\ell}}{\gamma_{1}}$,

where: $\omega^{*}=\frac{\omega}{\gamma_{3}}$

$\gamma_{1}=\frac{\alpha R_{0}^{2} I_{0}^{3} \ell}{\pi D^{2} \lambda}$

$\gamma_{3}=\frac{a}{\ell^{2}}$.

The amplitude of $U_{3 \omega}$ given by relation (54) depends on two adimensional numbers $\gamma_{2}$ and $\gamma_{4}$ with: 
$\gamma_{4}=\frac{4 R_{0} I_{0}^{2} \alpha \ell}{\pi D^{2} \lambda}$.

So that: $U_{3 \omega}^{n \ell *}=f_{n \ell}\left(\gamma_{2}, \gamma_{4}, \omega^{*}, t^{*}\right)$,

with: $U_{3 \omega}^{n \ell *}=\frac{U_{3 \omega}^{n \ell}}{\gamma_{1}}$

and: $t^{*}=\gamma_{3} t$.

When the sinusoidal periodic regime is reached, relation (62) becomes:

$U_{3 \omega}^{n \ell *}=f_{n \ell}\left(\gamma_{2}, \gamma_{4}, \omega^{*}\right)$.

The deviation between the linear and the non-linear models depends on the two parameters $\gamma_{2}$ and $\gamma_{4}$. Firstly, we will study the case $h=0$ corresponding to $\gamma_{2}=0$.

We have calculated the relative deviation between the tension $U_{3 \omega}^{\ell}$ calculated with the linear model and the tension $U_{3 \omega}^{n \ell}$ calculated by the non-linear model for a low frequency leading to the maximum deviation (cf. figure 5).

Table 3 presents the numerical results obtained for the various cases corresponding to quite different thermal properties, dimensions and temperature variations.

Figure 6 represents the maximum relative deviation $\left(U_{3 \omega}^{n \ell}-U_{3 \omega}^{\ell}\right) / U_{3 \omega}^{\ell}$ as a function of $\gamma_{4}$ for $\gamma_{2}=0(h=0)$.

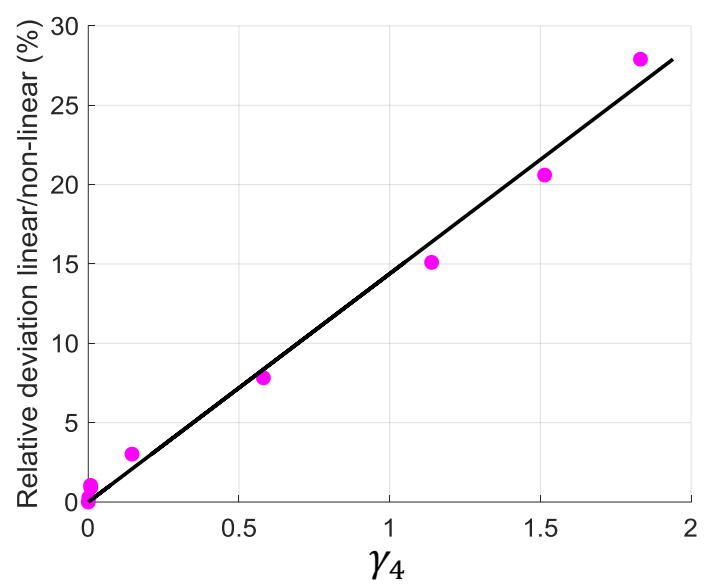

Figure 6: Relative deviation $\frac{U_{3 \omega}^{n \ell}-U_{3 \omega}^{\ell}}{U_{3 \omega}^{\ell}}(\%)$ of the maximum amplitude between the linear and the non-linear models versus $\gamma_{4}($ for $h=0)$ and linear regression

A linear regression leads to $: \frac{U_{3 \omega}^{n \ell}-U_{3 \omega}^{\ell}}{U_{3 \omega}^{\ell}}=14.31 \times \frac{4 R_{0} I_{0}^{2} \alpha \ell}{\pi D^{2} \lambda}$.

It enables to set a practical condition: if $\gamma_{4}<0.07$ then the deviation between the two models is lower than $1 \%$. Thus if $h=0$, the linear approximation is valid if:

$\frac{\rho_{0} I_{0}^{2} \alpha \ell^{2}}{D^{4} \lambda}<0.043$

One can notice that the deviation decreases for the highest frequencies, but in the same time the amplitude of the signal decreases (cf. figure 5) when the frequency increases so that the measurement is less precise. 
Figure 7 represents the relative deviation between the two models for a constant value of $\gamma_{4}$. A fairly good correlation of $\left(U_{3 \omega}^{n \ell}-U_{3 \omega}^{\ell}\right) / U_{3 \omega}^{\ell}$ as a function of $\gamma_{2}$ is given by:

$L C=\frac{14.4 \gamma_{4}}{1+0.17 \gamma_{2}{ }^{0.85}}$,

as shown by figure 8 where the values of $\left(U_{3 \omega}^{n \ell}-U_{3 \omega}^{\ell}\right) / U_{3 \omega}^{\ell}$ have been represented as a function of $L C$ for all the cases of table 3 . One can notice that all the points are close to the straight line $y=x$.

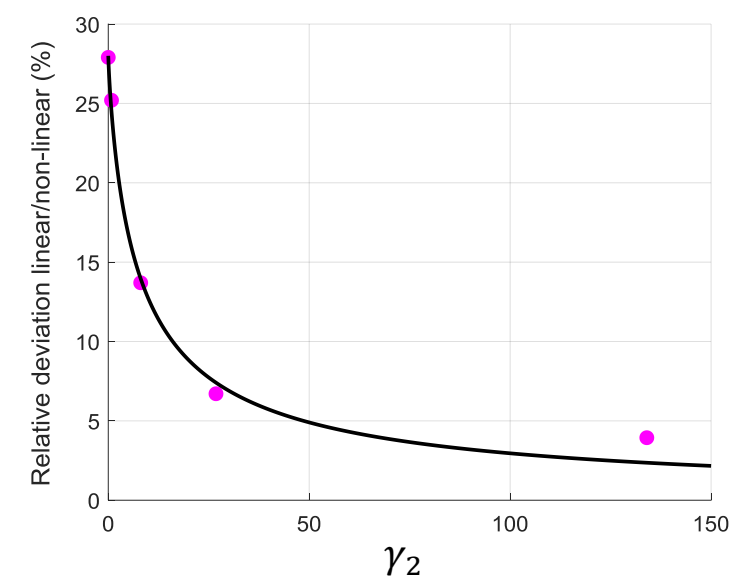

Figure 7: Relative deviation $\frac{U_{3 \omega}^{n \ell}-U_{3 \omega}^{\ell}}{U_{3 \omega}^{\ell}}(\%)$ of the maximum amplitude between the linear and the non-linear models versus $\gamma_{2}$ and a regression

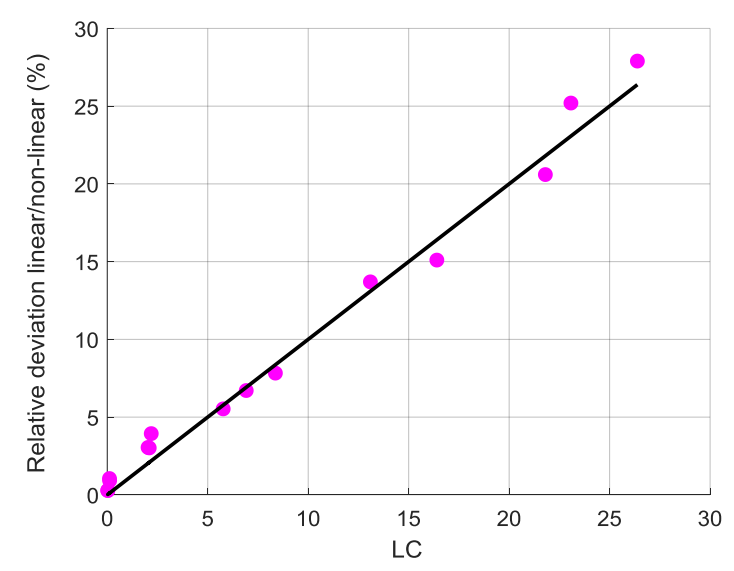

Figure 8: Relative deviation $\frac{U_{3 \omega}^{n \ell}-U_{3 \omega}^{\ell}}{U_{3 \omega}^{\ell}}(\%)$ of the maximum amplitude between the linear and the non-linear models versus the linearity criterion $L C=\frac{14.4 \gamma_{4}}{1+0.17 \gamma_{2}{ }^{0.85}}$

Figure 7 also shows that the relative deviation decreases when the heat transfer coefficient $h$ increases $\left(\gamma_{2}\right.$ is proportional to $\left.h\right)$. Nevertheless, the sensitivity of $U_{3 \omega}^{\ell}$ decreases when $h$ increases.

As an example, the amplitude of the third harmonic $U_{3 \omega}$ of the electrical tension $U$ has been measured by a lock-in amplifier for the configuration «Iron I ». Figure 9 shows a very good agreement between the experimental values and the theoretical values calculated by the non- 
linear model. This figure also highlights the deviation between the linear and the non-linear models.

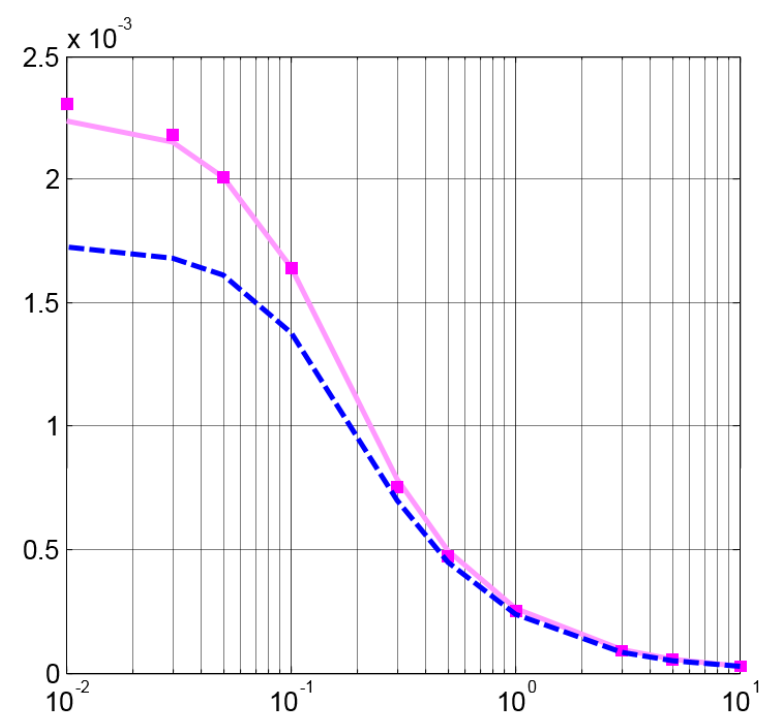

$\omega$

Figure 9: Amplitude of the electrical tension $U_{3 \omega}$ of an iron wire (Iron I) : experimental curve $(\square)$ and the linear (- -$)$ and non-linear (-) models simulations.

\section{Conclusion}

An analytical solution of the mean temperature rising of a wire during a $3 \omega$ experiment has been developed. This solution takes into account the non-linearity of the problem caused by the variation of the wire resistance during its heating. This solution enables the definition of a unique criterion to ensure the validity of the linear solution that can then be easily used for parameter estimation.

Moreover, it has been demonstrated that the connecting wires have no influence on the heat transfer if their diameters are greater than $1 \mathrm{~mm}$.

\section{Nomenclature}

a Thermal diffusivity

$K_{i} \quad$ Constants

c Specific heat

D Diameter

$h \quad$ Heat transfer coefficient

I Intensity

$I_{0} \quad$ Amplitude of the intensity $I$

$L C \quad$ Linearity criterion

$\ell \quad$ Length

$P \quad$ Heat flow rate

$p \quad$ Laplace parameter

$\dot{q} \quad$ Volume heat flow rate

$R_{0} \quad$ Initial electric resistance

$R \quad$ Electric resistance at a temperature $T$

$S \quad$ Section $\mathrm{m}^{2} \mathrm{~s}^{-1}$

$\mathrm{J} \mathrm{Kg}^{-1} \mathrm{~K}^{-1}$

$\mathrm{m}$

$\mathrm{W} \mathrm{m} \mathrm{m}^{-2} \mathrm{~K}^{-1}$

A

A

$\mathrm{m}$

W

$\mathrm{s}^{-1}$

$\mathrm{W} \mathrm{m}^{-3}$

$\Omega$

$\Omega$

$\mathrm{m}^{2}$ 


$\begin{array}{lll}t & \text { time } & \mathrm{s} \\ T & \text { Temperature } & \mathrm{K} \\ \bar{T} & \text { Mean temperature } & \mathrm{K} \\ U & \text { Electric potential difference } & \mathrm{V} \\ V & \text { Electric potential } & \mathrm{V} \\ \alpha & \text { Resistance temperature coefficient } & \mathrm{K}^{-1} \\ \Delta T & \text { Temperature difference } & \mathrm{K} \\ \gamma_{i} & \text { Constants } & \\ \lambda & \text { Thermal conductivity } & \mathrm{W} \mathrm{m}^{-1} \mathrm{~K}^{-1} \\ \psi & \text { Amplitude of the temperature rising } & \\ \rho & \text { Density } & \mathrm{kg} \mathrm{m}^{-3} \\ \rho_{0} & \text { Electric resistivity at } T_{\text {ext }} & \Omega \mathrm{m}^{-} \\ \theta & \text { Laplace transform of the temperature rising } & \\ \varphi & \text { Phase angle } & \mathrm{rd} \\ \omega & \text { Angular frequency } & \mathrm{s}^{-1} \\ & & \\ \text { Subscript } & \\ A C & \text { Periodic } & \\ D C & \text { Continuous } & \\ c & \text { Connection wire } & \\ e x t & \text { Ambient air } & \\ l & \text { linear } & \\ n l & \text { non-linear } & \\ 3 \omega & \text { Third harmonic } & \end{array}$

\section{References}

1. O.M. Corbino, Thermal oscillations in lamps of thin fibers with alternating current flowing through them and the resulting effect on the rectifier as a result of the presence of evennumbered harmonics, Phys. Z., 11 (1910) 413-417.

2. L.A. Rosenthal, Thermal response of bridewire used in electroexplosive devices, Rev. Sci. Instrum., 32 (9) (1961) 1033-1036.

3. L.R. Holland, Physical properties of Titanium III the specific heat, J. Appl. Phys., 34 (8) (1963) 2350-2357.

4. N.O. Birge, S.R. Nagel, Specific-heat spectroscopy of the glass transition, Phys. Rev. Lett., 544 (25) (1985) 2674-2677.

5. N.O. Birge, S.R. Nagel, Wide-frequency specific heat spectrometer, Rev. Sci. Instrum., 58 (8) (1987) 1464-1470.

6. N.O. Birge, P.K. Dixon, N. Menon, Specific heat spectroscopy: origins status and applications of the $3 \omega$ method, Thermochim. Acta, 304/305 (1997) 51-66.

7. R. Frank, V. Drach, J. Fricke, Determination of thermal conductivity and specific heat by

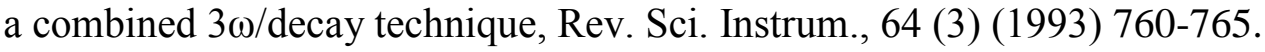

8. D.G. Cahill, Thermal conductivity measurement from 30 to $750 \mathrm{~K}$ : the $3 \omega$ method, Rev. Sci. Instrum., 61 (2) (1990) 802-808. 
9. D.G. Cahill, Thermal conductivity of thin films: measurements and understanding, Vacuum Sci. Technol. A, 7 (3) (1989) 1259-1266.

10. S.-M. Lee, D.G. Cahill, Heat transport in thin dielectric film, J. Appl. Phys., 81 (6) (1997) 2590-2595.

11. Lu L., Yi W., Zhang D.L., $3 \omega$ method for specific heat and thermal conductivity measurements, Review of Scientific Instruments, 72 (7) (2001) 2996-3003.

12. Dames C., Chen G., $1 \omega, 2 \omega$ and $3 \omega$ methods for measurements of thermal properties, Review of Scientific Instruments 76 (2005).

13. Wang Z.L., Tang D.W., Zhang W.G., Simultaneous measurements of the thermal conductivity, thermal capacity and thermal diffusivity of an individual carbon fiber, J. Phys. D: Appl. Phys. 40 (2007) 4686-4690.

14. Bhatta R. P., Annamalai S., Mohr R.K., Brandys M., Pegg I.L., Dutta B., High temperature thermal conductivity of platinium microwire by $3 \omega$ method, Review of Scientific Instruments 81 (2010).

15. Huzel, D., Reith, H., Schmitt, M.C., Picht, O., Müller, S., Toimil-Molares, M.E., Völklein, F., Characterization and Application of Thermoelectric Nanowires in NanowiresImplementations and applications, Chap. 14, In'Tech Editor, 2011.

16. Kimling J., Martens S., Nielsch K., Thermal conductivity measurements using $1 \omega$ and $3 \omega$ methods revisited for voltage-driven setups, Review of Scientific Instruments 82 (2011).

17. Choi T.Y., Poulikakos D., Tharian J., Sennhauser U., Measurement of thermal conductivity of individual multiwalled carbon nanotubes by the 3- $\omega$ method, Applied Physics Letter 87 (2005).

18. Bourgeois O., Fournier T., Chaussy J., Measurement of the thermal conductance of silicon nanowires at low temperatures, Journal of Applied Physics 101 (2007).

19. Moon J., Weaver K., Feng B., Chae H.G., Kumar S.Z. Balk J.B., Peterson G.P., Thermal conductivity measurement of individual poly (ether ketone) / carbon nanotube fibers using a steady-state dc thermal bridge method, Review of Scientific Instruments 83, (2012).

20. Depasse F., Grossel Ph., Trannoy N., Probe temperature and output voltage calculation for the SThM in AC mode, Superlattice Microst., 35 (2004) 315-322.

21. Xing C., Jensen C., Munro T., White B., Ban H., Chirtoc M., Thermal property characterization of fine fibers by the 3_omega technique, Appl. Therm. Eng., 71 (2014) 589-595.

22. Hou J., Wang X., Vellelacheruvu P., Guo J., Liu C., Cheng H.M., Thermal characterization of single wall carbon nanotube bundle using the self-heating $3 \omega$ technique, J. Appl. Phys., 100 (2006).

23. Dean G. Duffy, Green's functions with applications, CRC Press, 2001, ISBN-1-58488110-0. 